\title{
Purification
}

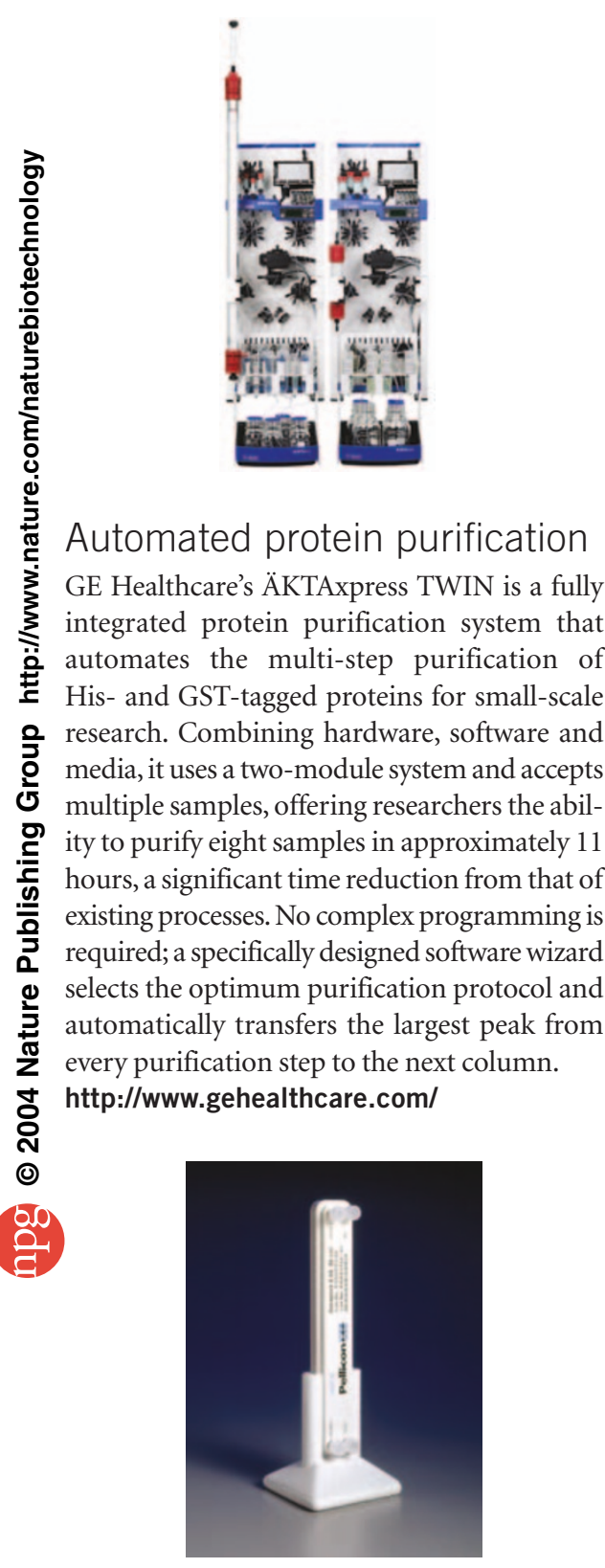

\section{Ultrafiltration device}

The Pellicon XL from Millipore incorporates tangential flow filtration technology to purify and concentrate from $300 \mathrm{ml}$ to 2 liters of solution down to a final volume of $20 \mathrm{ml}$. Self-contained, with Luer connections for easy setup, the device can be operated with either a peristaltic pump or with Millipore's Labscale TFF system. An optional graduated $100-\mathrm{ml}$ reservoir is also available for small-volume separations. Applications include cell harvesting and clarification, preparation of material for clini- cal trials, concentration and desalting of proteins, buffer exchange and depyrogenation. http://www.millipore.com/

\section{High-throughput purification}

HIS-Select iLAP (integrated Lysis and Affinity Purification) Plates from Sigma-Aldrich are coated with a proprietary high-density nickel chelate matrix that provides high-capacity affinity binding of recombinant fusion proteins with histidine-containing tags. An additional coating contains all of the reagents necessary for the lysis of bacterial cells for the purification of recombinant proteins. In addition, it includes protease inhibitors to help prevent the proteolytic breakdown of proteins. These two coatings allow bacterial culture to be added directly to the plate and simultaneously lyse cells and capture target protein. The ILAP plates may be used to confirm expression of the His-tagged target protein, protein-protein interaction assays and to rapidly screen multiple constructs. The resulting purified protein is compatible with protein assays, SDS-PAGE, western blot analysis and MALDI.

http://www.sigma-aldrich.com/

\section{mRNA purification kits}

Patent-pending peptide nucleic acid technology offers the highly efficient and specific capture of mRNA. The Poly T gripNA Probe found in Qbiogene's mTRAP mRNA isolation kits offers distinct advantages over oligo dT-based purification of mRNA. Ribosomal RNA and protein contamination is eliminated, traces of genomic DNA are reduced or completely removed and more representative mRNA populations can be purified.

http://www.qbiogene.com/

\section{RNA purification}

Gentra Systems' line of Versagene purification kits extracts RNA from tissue or cultured cells in as little as 20 minutes. Depending on sample type, yields range up to $600 \mu \mathrm{g}$ of pure, stable RNA with high integrity, suitable for downstream analysis or long-term storage. Kits come complete with pre-mixed reagents, and the entire purification process can be completed on the workbench at room temperature, without a fume hood. Hazardous disposal is not necessary, as the Versagene protocol does not employ harsh organic solvents, toxic reagents or reactive cyanide salts.

http://www.gentra.com/ 